\title{
INFLUENCE DE L'ÉLIMINATION DE LA CROUTE DANS L'ANALYSE DES FROMAGES
}

\author{
par \\ JEAN PIEN \\ Ingénieur Chimiste, Docteur ès Sciences \\ Directeur des Laboratoires de la Laiterie des Fermiers Réunis
}

Il est parfaitement connu que la croûte d'un fromage présente une composition différente de celle des parties sous-jacentes ou centrales. Deux causes principales interviennent pour créer ces différences :

$1^{\circ} \mathrm{La}$ dessiceation plus rapide et plus intense des couches superficielles ;

20 L'altération, sous l'influence des moisissures, des levures, des bactéries protéolytiques, etc., de la zone externe de certains fromages.

Ces deux causes produisent des effets variés suivant la nature et l'âge des fromages ; mais, d'une manière générale, on peut affirmer que le taux de matière grasse $\%$ de produit humide augmente plus vite dans la croûte que dans l'intérieur du fromage et, surtout, que le taux de matière grasse $\%$ de produit see - qui devrait rester constant si, seul, le taux d'humidité variait - augmente à son tour par suite d'une disparition progressive de la matière sèche ellemême, cet $^{+} e$ destruction étant le fait de l'activité des microorganismes de la croûte de certains fromages.

Il résulte de ce jeu de circonstances que le chiffre obtenu dans le dosage de la matière grasse et exprimé en \% de produit sec est différent suivant que l'on incorpore ou non la croûte dans la masse totale de l'échantillon à analyser. Comme l'analyse doit refléter la composition centésimale du fromage telle qu'elle découle des conditions de la fabrication (richesse du lait notamment) il était normal que l'on convînt de soustraire l'analyse à l'influence perturbatrice de la croûte, en éliminant celle-ci purement et simplement. C'est cette préoccupation que l'on retrouve dans un certain nombre de textes officiels relatifs à l'analyse des fromages et en particulier dans la Convention Internationale de Rome (26 av́ril 1934).

Mais, depuis cette époque, des éléments nouveaux sont intervenus dans la définition et l'analyse des fromages. Alors qu'autrefois on se bornait à définir un fromage par le taux de matière grasse exprimé en \% de produit sec (et dans certains cas par les dimensions et le pourcentage d'humidité) on a maintenant décidé de tenir compte pour certains d'entre eux du taux de matière grasse totale 
et du taux de matière sèche totale. On fait done intervenir le poids du fromage au moment de l'analyse (décret du 4 décembre 1941).

Dans cette nouv lle manière de faire, l'incidence de la composition particulière $i$ li croûte se présente sous un jour différent.

La croûte se dessèche et fait fléchir le poids du fromage.

Il en résulte une possibilité d'erreur dans le calcul de la matière grasse totale du fromage. Si, en effet, le taux de matière grasse \% de produit humide est déterminé seulement sur l'intérieur du fromage écroûté et si le chiffre ainsi obtenu est multiplié par le poids du fromage amoindri par la dessiccation de la croûte, le résultat final sera inférieur à la réalité. Il faudrait, pourrétablir la situation, ou bien utiliser le poids initial du fromage ou bien tenir compte de l'enrichissement relatif de la croûte en matière grasse c'est-à-dire, finalement, faire entrer celle-ci dans l'analyse de l'échantillon.

Même raisonnement en ce qui concerne le taux de matière sèche totale du fromage. Si l'analyse ne porte que sur les couches centrales et si le chiffre trouvé est rapporté au fromage entier dont le poids total a diminué par suite de la dessiccation de la croûte, le taux de matière sèche totale ainsi calculé est trop faible.

Done la considération des éléments totaux d'un fromage (matière grasse totale et matière sèche totale) implique qu'il doit être tenu compte de la composition particulière de la croûte dans l'ana. lyse de l'échantillon et la meilleure manière de résoudre cette question consiste évidemment à incorporer intimement cette croûte à l'échantillon entier au moment de l'analyse contrairement à ce qui était recommandé autrefois. Ce sont là précisément les raisons pour lesquelles M. le Directeur du Service de la Répression des Fraudes a cru devoir prescrire aux Laboratoires officiels de ne plus procéder à l'élimination de la croûte des fromages en vue de l'analyse (lettre du 31 juillet 1942).

Vo'ci un extrait de ce document :

“... J'ai l'honneur de vous faire connaître que l'écroûtage " qui avait sa raison d'être lorsqu'il s'agissait uniquement (pour "l'application du décret du 20 octobre 1936) de déterminer le "pourcentage de matière grasse rapportée à la matière sèche, ne " doit plus être pratiqué quand il s'agit de déterminer l'extrait sec "total pour l'application du décret du 4 décembre 1941."

$$
\text { *** }
$$

La question est donc entendue et il pourrait sembler superflu d'y insister davantage.

Il nous a cependant paru intéressant et utile d'y revenir, d'abord pour montrer les différences de composition qui peuvent 
exister entre les parties superficielles et internes des fromages, ensuite pour mettre en évidence l'erreur que l'on commettait jadis en éliminant la croûte de l'échantillon à analyser. Cette erreur apparaîtra dans la comparaison des chiffres (rapportés au poids du fromage entier) obtenus dans l'analyse d'une portion de ce fromage "croûte comprise» et dans l'analyse d'une autre portion effectuée sur l'intérieur seul.

\section{Techniques employées.}

Nous avons travaillé sur deux types de fromages :

10 Fromages à pâte molle (fromages moisis, tels que Camembert, Brie...);

$2^{\circ}$ Fromages à pâte ferme (fromages pressés tels que Port-Salut).

Dans les deux cas, nous avons examiné des fromages frais, des fromages demi-affinés et des fromages affinés.

Les essais ont été menés de la manière suivante :

Sur une portion du fromage on procédait à l'élimination complète de la eroûte qui était analysée à part. On analysait d'autre part la partie interne de cette même portion.

D'autre part, sur une autre portion de même poids (prélevée sur le même fromage) on réalisait un broyage parfait de l'échantillon en y incorporant la croûte : simple malaxage au mortier pour les pâtes molles, passage à la râpe mécanique pour les pâtes fermes. L'analyse de cet échantillon total était effectuée et comparée à celle de la partie interne.

Enfin les éléments totaux du fromage étaient calculés à l'aide des deux analyses précédentes et comparés entre eux. (Dans certains cas nous n'avions à notre disposition que des portions de fromages et non des fromages entiers. C'est pourquoi certains chiffres d'éléments "totaux" paraîtront anormaux. Néanmoins la comparaison entre les deux séries de résultats reste possible et justifiée.)

\section{$* * *$}




\section{PREMIÈRE PARTIE}

\section{COMPOSITION RESPECTIVE DE LA CROUTE ET DE L'INTÉRIEUR DES FROMAGES}

\section{CAS DES FROMAGes A PATE MOLLE (Camembert, Brie, Coulommiers, etc.)}

$1^{0}$ Fromages frais (quelques jours après la fabrication)..

\begin{tabular}{|c|c|c|c|c|c|c|c|c|c|}
\hline \multirow[t]{2}{*}{ (3) } & & \multicolumn{2}{|c|}{ Humidits \% } & \multicolumn{2}{|c|}{$\begin{array}{c}\text { Matière sèche } \\
\% \\
\end{array}$} & \multicolumn{2}{|c|}{$\begin{array}{c}\text { Matière grasse } \\
\% \text { de frais } \\
\end{array}$} & \multicolumn{2}{|c|}{$\begin{array}{c}\text { Matière grasse } \\
\% \text { de sec } \\
\end{array}$} \\
\hline & & croûte & $\begin{array}{l}\text { inté- } \\
\text { rieur }\end{array}$ & croûte & $\begin{array}{l}\text { inté. } \\
\text { rieur }\end{array}$ & croûte & $\begin{array}{l}\text { inté. } \\
\text { rieur }\end{array}$ & croûte. & $\begin{array}{l}\text { intes. } \\
\text { rieur }\end{array}$ \\
\hline 1 & $\ldots \ldots \ldots \ldots \ldots$ & 53,75 & 55,65 & 46,25 & 45,35 & 16,43 & 15,94 & 35,52 & 35,94 \\
\hline 2 & $\ldots \ldots \ldots \ldots$ & 60,10 & 66,50 & 39,90 & 33,50 & 12,88 & 9,91 & 32,28 & 29,58 \\
\hline 3 & $\ldots, \ldots \ldots \ldots$ & 53,31 & 61,16 & 46,69 & 38,84 & 14,26 & 11,90 & 30,54 & 30,63 \\
\hline 4 & $\ldots \ldots \ldots \ldots$ & 60,05 & 49,30 & 39,95 & 50,70 & 1,37 & 1,55 & 3,42 & 3,05 \\
\hline 5 & $\ldots \ldots \ldots \ldots \ldots$ & 59,22 & 61,71 & 40,78 & 38,29 & 14,92 & 13,37 & 36,58 & 34,91 \\
\hline 6 & $\ldots \ldots \ldots \ldots$ & 55,84 & 61,60 & 44,16 & 38,40 & 17,94 & 14,76 & 40,62 & 38,43 \\
\hline 7 & $\ldots \ldots \ldots \ldots$ & 54,10 & 56,35 & 45,90 & 43,65 & 12,47 & 13,44 & 27,16 & $\dot{5} 0,78$ \\
\hline 8 & $\ldots \ldots \ldots \ldots$ & 51,20 & 63,40 & 48,80 & 36,60 & 18,89 & 17,84 & 38,70 & 48,74 \\
\hline 9 & $\ldots \ldots \ldots \ldots$ & 65,17 & 65,08 & 34,83 & 34,92 & 0,58 & 1,09 & 1,66 & 3,12 \\
\hline 10 & $\ldots \ldots$ & 60,13 & 63,94 & 39,87 & 36,06 & 2,99 & 2,68 & 7,52 & 7,43 \\
\hline 11 & . & 52,10 & 52,51 & 47,90 & 47,49 & 15,68 & 17,62 & 32,69 & 37,10 \\
\hline 12 & . & 57,43 & 53,18 & $4 \div, 57$ & 46,82 & 13,50 & 18,91 & 31,71 & 40,38 \\
\hline 13 & & 66,68 & 73,84 & 33,32 & 26,16 & 0,84 & 1,74 & 2,52 & 6,65 \\
\hline 14 & & 56,30 & 50,93 & 43,70 & 49,07 & 15,81 & 19,94 & 36,17 & 40,63 \\
\hline 15 & & 67,56 & 64,23 & 32,44 & 35,77 & 0,61 & 0,91 & 1,88 & 2,54 \\
\hline 16 & $\ldots \ldots \ldots \ldots$ & 65,89 & 64,13 & 34,11 & 35,87 & 0,77 & 0,79 & $2,2 \div 5$ & 2,20 \\
\hline
\end{tabular}

\section{Commentaires}

a) En ce qui concerne l'humidité. Dans dix cas sur seize la surface était plus sèche que l'intérieur. Mais dans six cas elle était plus humide. Les écarts sont importants ;

b) En ce qui concerne la richesse en matière grasse sur sec. Dans cinq cas sur seize il y avait identité entre les riohesses de la surface et de l'intérieur. Dans huit cas la surface était moins grasse et parfois de beaucoup (jusqu'à 10 grammes pour 100 grammes de matière sèche) que l'intérieur. Dans trois cas, elle était un peu plus grasse (2 à $3 \%$ au maximum).

Donc, sur des fromages à pâte molle frais, la surface est généralement plus sèche et généralement moins grasse (parfois de beaucoup) que l'intérieur. Les écarts d'humidité entre surface et intérieur sont parfois considérables. 


\section{$2^{\circ}$ Fromages demi-affinés.}

\begin{tabular}{|c|c|c|c|c|c|c|c|c|c|}
\hline & & \multicolumn{2}{|c|}{ Humidité \% } & \multicolumn{2}{|c|}{$\begin{array}{c}\text { Matière sèche } \\
\%\end{array}$} & \multicolumn{2}{|c|}{$\begin{array}{c}\text { Matière grasse } \\
\% \text { de frais }\end{array}$} & \multicolumn{2}{|c|}{$\begin{array}{c}\text { Matière grasse } \\
\% \text { de sec }\end{array}$} \\
\hline & & croûte & $\begin{array}{l}\text { inté. } \\
\text { rieur }\end{array}$ & eroûte & $\begin{array}{l}\text { inté- } \\
\text { rieur }\end{array}$ & eroûte & $\begin{array}{l}\text { ints. } \\
\text { rieur }\end{array}$ & eroûte & $\begin{array}{l}\text { inté- } \\
\text { rieur }\end{array}$ \\
\hline 1 & $\ldots \ldots \ldots \ldots \ldots$ & 63,79 & 59,74 & 36,21 & 40,26 & 0,93 & 1,58 & 2,56 & 3,92 \\
\hline$z$ & $\ldots \ldots \ldots \ldots \ldots$ & 56,74 & 56,00 & 43,20 & 44,00 & 9,79 & 14,27 & 22,58 & 32,43 \\
\hline 3 & . & 53,61 & 51,06 & 46,39 & 48,94 & 14,66 & 17,23 & 31,60 & 35,20 \\
\hline 4 & $\ldots \ldots \ldots \ldots \ldots$ & 59,45 & 53,61 & 40,55 & 46,39 & 14,58 & 17,29 & 35,95 & 37,27 \\
\hline 5 & $\ldots \ldots \ldots \ldots \ldots$ & 63,50 & 65,31 & 36,50 & 34,69 & 0,28 & 0,23 & 0,76 & 0,66 \\
\hline 6 & $\ldots$ & 59,83 & 62,22 & 40,17 & 37,78 & 11,29 & 10,24 & 28,10 & 27,10 \\
\hline 7 & $\ldots \ldots \ldots \ldots \ldots$ & 55,41 & 51,41 & 44,59 & 48,59 & 15,04 & 16,25 & 33,73 & 33,44 \\
\hline 8 & $\ldots \ldots$ & 57,73 & 57,46 & 42,27 & 42,54 & 14,66 & 16,52 & 34,68 & 38,83 \\
\hline 9 & $\ldots \ldots \ldots \ldots \ldots$ & 55,11 & 52,93 & 44,89 & 47,07 & 15,14 & 17,27 & 33,72 & 36,69 \\
\hline 10 & $\ldots$ & 51,36 & 50,02 & 48,64 & 49,98 & 16,22 & 19,56 & 33,34 & 39,13 \\
\hline 11 & $\ldots$ & 46,19 & 51,74 & 53,81 & 48,26 & 19,88 & 18,08 & 36,88 & 37,46 \\
\hline 12 & $\ldots \ldots \ldots \ldots \ldots$ & 51,76 & 55,54 & 48,24 & 44,46 & 16,40 & 16,52 & 33,99 & 37,15 \\
\hline
\end{tabular}

\section{Commentaires}

a) En ce qui concerne l'humidité. Dans un tiers des cas, la surface est plus sèche que l'intérieur; dans deux tiers des cas, elle est plus humide. Les écarts sont beaucoup moins sensibles que sur les fromages frais;

b) En ce qui concerne la richesse en matière grasse sur sec. Dans un tiers des cas il y a identité (à $1 \%$ près) entre la richesse de la surface et celle de l'intérieur. Dans tous les autres cas, la surface est moins riche en matière grasse que l'intérieur (parfois de beaucoup jusqu'à 10 grammes d'écart pour 100 grammes de matière sèche).

\section{$3^{\circ}$ Fromages affinés.}

\begin{tabular}{|c|c|c|c|c|c|c|c|c|c|}
\hline & & \multicolumn{2}{|c|}{ Humídité \% } & \multicolumn{2}{|c|}{$\begin{array}{c}\text { Matière sèche } \\
\% \\
\end{array}$} & \multicolumn{2}{|c|}{$\begin{array}{c}\text { Matière grasse } \\
\% \text { de frais } \\
\end{array}$} & \multicolumn{2}{|c|}{$\begin{array}{c}\text { Matière grasse } \\
\% \text { de sec }\end{array}$} \\
\hline & & eroûte & $\begin{array}{l}\text { inte- } \\
\text { rieur }\end{array}$ & croûte & $\begin{array}{l}\text { inté- } \\
\text { rieur }\end{array}$ & croûte & $\begin{array}{l}\text { inté. } \\
\text { rieur }\end{array}$ & eroûte & $\begin{array}{l}\text { inté- } \\
\text { riour }\end{array}$ \\
\hline 1 & , & 53,89 & 57,11 & 46,11 & 42,89 & 1,76 & 1,61 & 3,81 & 3,75 \\
\hline 2 & $\ldots \ldots \ldots \ldots \ldots$ & 56,81 & 57,84 & 43,19 & 42,16 & 17,88 & 13,71 & 41,39 & 32,51 \\
\hline 3 & $\ldots \ldots \ldots \ldots \ldots$ & 54,32 & 60,87 & 45,68 & 39,13 & 0,76 & 0,42 & 1,66 & 1,07 \\
\hline 4 & $\ldots \ldots \ldots \ldots \ldots$ & 50,44 & 53,93 & 49,56 & 46,07 & 19,10 & 16,60 & 38,53 & 36,03 \\
\hline 5 & $\ldots \ldots \ldots \ldots \ldots$ & 57,86 & 59,42 & 42,14 & 40,58 & 13,76 & 12,28 & 32,65 & 30,26 \\
\hline 6 & $\ldots \ldots \ldots \ldots \ldots$ & 38,97 & 53,43 & 61,03 & 46,57 & 23,29 & 15,90 & 38,16 & 33,92 \\
\hline 7 & $\ldots \ldots \ldots \ldots \ldots$ & 52,72 & 55,32 & 47,28 & 44,68 & 17,87 & 15,26 & 37,79 & 34,15 \\
\hline 8 & $\ldots \ldots \ldots \ldots$ & 44,97 & 49,86 & 55,03 & 50,14 & 22,45 & 20,43 & 40,79 & 40,74 \\
\hline
\end{tabular}




\section{Commentaires}

a) En ce qui concerne l'humidité. Dans tous les cas la surface est plus sèche que l'intérieur ;

b) En ce qui concerne la teneur en matière grasse sur sec. Dans tous les cas la surface est plus riche que l'intérieur ou d'une richesse au moins égale. Il y a parfois de grands écarts (jusqu'à 9 grammes).

\section{Conclusions en ce qui concerne les pâtes molles}

\section{Humidité.}

Quand les fromages sont jeunes la surface est tantôt plus sèche, tantôt plus humide que l'intérieur. Les écarts sont d'abord très importants. Ils s'atténuent ensuite peu à peu.

Sur les fromages affinés, la croûte est toujours plus sèche que l'intérieur.

\section{Richesse en matière grasse sur sec.}

Dans les fromages frais ou demi-affinés la surface est souvent moins grasse que l'intérieur (parfois de beaucoup). La matière grasse superficielle est sans doute partiellement brûlée par les moisissures.

En revanche, sur les fromages entièrement affinés c'est l'inverse que nous constatons : la croûte est plus riche en matière grasse que l'intérieur ou au moins de richesse égale. Il est facile de s'expliquer ce phénomène en se rappelant qu'au cours de l'affinage les fromages subissent des déperditions de matière sèche (dégradation de la matière azotée sous forme de produits volatils) qui entraînent un enrichissement relatif en matière grasse (1): Or, sur la croûte, cette dégradation est plus intense qu'à l'intérieur dans le cas des fromages à pâte molle. L'enrichissement apparent est donc plus important sur la croûte qu'à l'intérieur dans les fromages âgés et c'est pourquoi la croûte est relativement plus riche en matière grasse en général.

\section{CAS DES FROMAGES A PATE FERME (Port-Salut, Edam, etc.)}

$1^{\circ}$ Fromages frais (quelques jours après la fabrication).

(1) Voir notręe étude de cette question : Modifications dans la composition dés fromages au cours de leur conservation. Le Lait, 1937, page 1040. 


\begin{tabular}{|c|c|c|c|c|c|c|c|c|c|}
\hline \multirow{2}{*}{\multicolumn{2}{|c|}{$x=$}} & \multicolumn{2}{|c|}{ Humidité \% } & \multicolumn{2}{|c|}{$\begin{array}{c}\text { Matière sèche } \\
\%\end{array}$} & \multicolumn{2}{|c|}{$\begin{array}{c}\text { Matière grasse } \\
\% \text { de frais }\end{array}$} & \multicolumn{2}{|c|}{$\begin{array}{c}\text { Matière grasse } \\
\% \text { de sec }\end{array}$} \\
\hline & & eroûte & $\begin{array}{l}\text { inté- } \\
\text { rieur }\end{array}$ & croûte & $\begin{array}{l}\text { inté, } \\
\text { rieur }\end{array}$ & eroûte & $\begin{array}{l}\text { inte. } \\
\text { rieur }\end{array}$ & eroûte & $\begin{array}{l}\text { inté. } \\
\text { rieur }\end{array}$ \\
\hline 1 & . & 34,92 & 47,93 & 65,08 & 52,07 & 16,31 & 7,36 & 25,06 & 14,13 \\
\hline 2 & & 38,47 & 46,30 & 61,53 & 53,70 & 22,17 & 16,50 & 36,03 & 30,72 \\
\hline 3 & $\ldots \ldots \ldots \ldots \ldots$ & 40,86 & 45,42 & 59,14 & 54,58 & 20,16 & 19,81 & 34,09 & 36,29 \\
\hline 4 & $\ldots \ldots \ldots \ldots \ldots$ & 50,70 & 55,97 & 49,30 & 44,03 & 8,01 & 6,21 & 16,24 & 14,10 \\
\hline 5 & & 38,77 & 49,13 & 61,23 & 50,87 & 20,85 & 18,03 & 36,01 & 35,44 \\
\hline 6 & $\cdots$ & 25,70 & 46,15 & 74,30 & 53,85 & 28,11 & 20,50 & 37,83 & 38,06 \\
\hline 7 & $\ldots \ldots \ldots \ldots \ldots$ & 49,47 & 55,31 & 50,53 & 44,69 & 12,80 & 11,37 & 25,33 & 25,66 \\
\hline 8 & $\ldots \ldots \ldots \ldots \ldots$ & 35,39 & 47,49 & 64,61 & 52,51 & 21,46 & 17,12 & 33,21 & 32,64 \\
\hline 9 & $\ldots \ldots \ldots$ & 37,73 & 47,93 & 62,27 & 52,07 & 21,21 & 17,50 & 34,06 & 33,60 \\
\hline
\end{tabular}

\section{Commentaires}

a) En ce qui concerne l'humidité. Dans tous les cas la croûte est moins humide que l'intérieur. Les écarts sont parfois très notables ;

-b) En ce qui concerne la richesse en matière grasse sur sec. Si l'on excepte un seul cas où la croûte était un peu moins riche que l'intérieur (d'environ $2 \%$ ), dans tous les autres cas elle était ou plus riche $(50 \%$ des cas) ou de richesse égale.

\section{$2^{\circ}$ Fromages demi-affinés.}

\begin{tabular}{|c|c|c|c|c|c|c|c|c|c|}
\hline & & \multicolumn{2}{|c|}{ Humidité \% } & \multicolumn{2}{|c|}{$\begin{array}{c}\text { Matière sèche } \\
\%\end{array}$} & \multicolumn{2}{|c|}{$\begin{array}{c}\text { Matière grases } \\
\% \text { de frais }\end{array}$} & \multicolumn{2}{|c|}{$\begin{array}{c}\text { Matière grasse } \\
\% \text { de sec }\end{array}$} \\
\hline & & croûte & $\begin{array}{l}\text { inté. } \\
\text { rieur }\end{array}$ & croûte & $\begin{array}{l}\text { inté. } \\
\text { rieur }\end{array}$ & croûte & $\begin{array}{l}\text { inté- } \\
\text { rieur }\end{array}$ & croûte & $\begin{array}{l}\text { inté- } \\
\text { rieur }\end{array}$ \\
\hline 1 & $\ldots \ldots \ldots \ldots \ldots$ & 38,45 & 55,46 & 61,55 & 44,54 & 11,45 & 8,76 & 18,60 & 19,66 \\
\hline 2 & $\ldots \ldots \ldots \ldots \ldots$ & 44,29 & 56,50 & 55,71 & 43,50 & 11,40 & 9,74 & 20,46 & 22,38 \\
\hline 3 & $\ldots \ldots \ldots \ldots$ & 35,06 & 47,26 & 64,94 & 52,74 & 20,69 & 16,96 & 31,86 & 32,15 \\
\hline 4 & $\ldots \ldots \ldots$ & 41,28 & 55,56 & 58,72 & 44,44 & 11,74 & 6,83 & 19,99 & 15,34 \\
\hline 5 & $\ldots \ldots \ldots \ldots$ & 43,03 & 53,90 & 56,97 & 46,10 & 13,68 & 8,42 & 24,01 & 18,26 \\
\hline 6 & ............ & 43,71 & 56,68 & 56,29 & 43,32 & 10,14 & 7,83 & 18,01 & 18,07 \\
\hline 7 & $\ldots \ldots$ & 45,76 & 57,26 & 54,24 & 42,74 & 13,09 & 9,75 & 24,13 & 22,81 \\
\hline 8 & $\ldots \ldots \ldots$ & 51,67 & 53,96 & 48,33 & 46,04 & 10,67 & 10,58 & 22,07 & 22,98 \\
\hline 9 & n.......... & 37,10 & 48,00 & 62,90 & 52,00 & 20,40 & 16,73 & 32,43 & 32,17 \\
\hline 10 & $\ldots \ldots \ldots \ldots \ldots$ & 49,44 & 54,82 & 50,56 & 45,18 & 11,81 & 9,97 & 23,35 & 22,06 \\
\hline 11 & & 49,49 & 54,07 & 50,51 & 45,93 & 12,56 & 9,91 & 24,86 & 21,57 \\
\hline 12 & n......... & 47,87 & 58,38 & 52,13 & 41,62 & 14,44 & 9,24 & 26,54 & 22,20 \\
\hline 13 & n.......... & 47,77 & 57,90 & 52,23 & 42,10 & 11,11 & 8,65 & 21,27 & 20,54 \\
\hline
\end{tabular}




\section{Commentaires}

a) En ce qui concerne l'humidité. Dans tous les cas la croûte est plus sèche que l'intérieur. Les écarts sont parfois très notables;

b) En ce qui concerne la teneur en matière grasse sur sec. Si l'on excepte deux cas où la croûte était un peu moins riche que l'intérieur (de 1 à $2 \%$ ) dans tous les autres cas elle était ou plus riche (plus de la moitié des cas) ou de richesse égale. Les écarts étant parfois très notables.

\section{$3^{\circ}$ Fromages affinés.}

\begin{tabular}{|c|c|c|c|c|c|c|c|c|c|}
\hline & & \multicolumn{2}{|c|}{ Humidité \% } & \multicolumn{2}{|c|}{$\begin{array}{c}\text { Matière sèche } \\
\%\end{array}$} & \multicolumn{2}{|c|}{$\begin{array}{c}\text { Matière grasse } \\
\% \text { de frais }\end{array}$} & \multicolumn{2}{|c|}{$\begin{array}{c}\text { Matière grasse } \\
\% \text { de sec }\end{array}$} \\
\hline & & croûte & $\begin{array}{l}\text { inté- } \\
\text { rieur }\end{array}$ & croûte & $\begin{array}{l}\text { inté. } \\
\text { rieur }\end{array}$ & croûte & $\begin{array}{l}\text { inté- } \\
\text { rieur }\end{array}$ & croûte & $\begin{array}{l}\text { inté. } \\
\text { riour }\end{array}$ \\
\hline 1 & $\ldots \ldots \ldots \ldots \ldots$ & 35,56 & 51,70 & 64,44 & 48,30 & 13,87 & 9,63 & 21,52 & 19,91 \\
\hline 2 & $\ldots \ldots \ldots \ldots$ & 36,91 & 53,36 & 63,09 & 46,64 & 14,50 & 11,06 & 22,98 & 23,71 \\
\hline 3 & $\ldots \ldots \ldots \ldots \ldots$ & 27,75 & 43,11 & 72,25 & 56,89 & 28,32 & 21,97 & 39,16 & 38,61 \\
\hline 4 & n......... & 27,60 & 55,08 & 72,40 & 44,92 & 17,10 & 11,50 & 23,47 & 25,60 \\
\hline 5 & $\ldots \ldots \ldots \ldots \ldots$ & 28,46 & 44,81 & 71,54 & 55,19 & 17,78 & 13,75 & 24,85 & 24,91 \\
\hline 6 & ............ & 33,59 & 53,59 & 66,41 & 46,41 & 15,33 & 11,62 & 23,09 & 25,03 \\
\hline 7 & n........ & 40,35 & 55,02 & 59,65 & 44,98 & 10,81 & 6,43 & 18,12 & 14,29 \\
\hline 8 & $\ldots \ldots \ldots \ldots \ldots$ & 27,42 & 38,29 & 72,58 & 61,71 & 14,36 & 11,04 & 19,78 & 17,89 \\
\hline 9 & $\ldots \ldots \ldots \ldots \ldots$ & 38,13 & 55,73 & 61,87 & 44,27 & 9,32 & 5,10 & 15,06 & 11,52 \\
\hline 10 & …....... & 45,22 & 56,15 & 54,78 & 43,85 & 11,30 & 7,23 & 20,62 & 16,48 \\
\hline 11 & $\ldots \ldots \ldots \ldots \ldots$ & 23,78 & 50,67 & 76,22 & 49,33 & 17,56 & 9,76 & 23,03 & 19,78 \\
\hline 12 & $\ldots \ldots \ldots \ldots$ & 22,64 & 41,78 & 77,36 & 58,22 & 21,32 & 14,37 & 27,55 & 24,68 \\
\hline 13 & n.......... & 25,21 & 42,41 & 74,79 & 57,59 & 28,98 & 22,47 & 38,74 & 39,01 \\
\hline 14 & $\ldots \ldots \ldots \ldots \ldots$ & 42,96 & 51,83 & 57,04 & 48,17 & 19,83 & 15,75 & 34,76 & 32,69 \\
\hline 15 & $\ldots \ldots \ldots \ldots$ & 38,34 & 55,41 & 61,66 & 44,59 & 12,51 & 7,72 & 20,28 & 17,31 \\
\hline 16 & $\ldots \ldots \ldots \ldots \ldots$ & 26,46 & 48,82 & 73,54 & 51,18 & 16,74 & 10,71 & 22,76 & 20,92 \\
\hline
\end{tabular}

\section{Commentaires}

a) En ce qui concerne l'humidité. Dans tous les cas la croûte est beaucoup plus sèche que l'intérieur. Il y a des écarts très considérables atteignant le simple au double et parfois même le dépassant ;

b) En ce qui concerne la teneur en matière grasse sur sec. Si l'on excepte trois cas où la crô̂te était un peu moins riche que l'intérieur, dans tous les autres eas elle etait plus riche ou de richesse égàle. 


\section{Conclusions en ce qui concerne les pâtes fermes}

\section{Humidité.}

Quel que soit l'âge du fromage, la croûte est toujours plus sèche que l'intérieur, même dans les fromages très jeunes. Les écarts sont parfois considérables surtout dans les fromages affinés.

\section{Richesse en matière grasse.}

Dans la majorité des cas, la croûte est plus riche en matière grasse que l'intérieur quel que soit l'âge du fromage. Dans quelques cas il y a égalité de richesse. Exceptionnellement la croûte est un peu moins riche que l'intérieur. L'âge du fromage ne change rien à cette règle qui paraît générale.

L'explication de cet enrichissement de la croûte peut être la même que celle que nous avons suggérée pour les pâtes molles - perte de matière sèche dans les parties superficielles par suite d'une dégradation partielle des matières azotées, d'où enrichissement relatif en matière grasse.

\section{$*^{*} *$}

\section{DEUXIÈME PARTIE}

\section{INFLUENCE DE L'ELIMINATION DE LA CROUTE SUR LES RESULTATS DE L'ANALYSE}

Les conclusions précédentes nous permettent de prévoir que les résultats de l'analyse seront en général différents suivant qu'elle aura été effectuée sur le fromage écroûté (comme on le faisait autrefois) ou sur le fromage entier, croûte comprise.

L'expérience confirme bien cette prévision. Nous donnons cidessous le résultat de nos essais dans les divers cas envisagés cidessus, en nous bornant à reproduire les taux d'humidité pour cent et de matière grasse pour cent de matière sèche mais en y ajoutant les éléments totaux des fromages calculés dans les deux cas. La comparaison des deux groupes de chiffres montrera l'erreur que l'on commettait en n'incorporant pas la croûte dans l'échantillon destiné à l'analyse.

\section{CAS DES FROMAGES A PATE MOLLE (Camembert, Coulommiers, Brie, eto.)}

10 Fromages frais (quelques jours après la fabrication). 


\begin{tabular}{|c|c|c|c|c|c|c|c|c|}
\hline & \multicolumn{2}{|c|}{ Humidité \% } & \multicolumn{2}{|c|}{$\begin{array}{c}\text { Matière grasse } \\
\% \text { de sec } \\
\end{array}$} & \multicolumn{2}{|c|}{$\begin{array}{c}\text { Matière grasse } \\
\text { totale } \\
\end{array}$} & \multicolumn{2}{|c|}{$\begin{array}{c}\text { Matière sèche } \\
\text { totale }\end{array}$} \\
\hline & \begin{tabular}{|c|} 
avec \\
eroûte
\end{tabular} & $\begin{array}{l}\text { sans } \\
\text { croûte }\end{array}$ & $\begin{array}{l}\text { avec } \\
\text { croûte }\end{array}$ & $\begin{array}{c}\text { sans } \\
\text { croûte }\end{array}$ & $\begin{array}{l}\text { avec } \\
\text { croûte }\end{array}$ & $\begin{array}{c}\text { sans } \\
\text { croûte }\end{array}$ & \begin{tabular}{|c|} 
avec \\
croûte
\end{tabular} & $\begin{array}{l}\text { sans } \\
\text { croûte }\end{array}$ \\
\hline 1 & 56,40 & 55,65 & 35,18 & 35,94 & 36,81 & 38,25 & 104,64 & 106,44 \\
\hline $2 \ldots \ldots$ & 63,15 & 66,50 & 29,33 & 29,58 & 38,05 & 34,88 & 129,71 & 117,92 \\
\hline $3 \ldots \ldots \ldots$ & 59,71 & 61,16 & 29,58 & 30,63 & 23,84 & 23,80 & 80,58 & 77,68 \\
\hline $4 \ldots \ldots \ldots$ & 58,94 & 49,30 & 4,48 & 3,05 & 6,51 & 5,48 & 145,35 & 179,47 \\
\hline 5 & 61,04 & 61,71 & 36,80 & 34,91 & 43,02 & 40,11 & 116,88 & 114,87 \\
\hline 6 & 59,40 & 61,60 & 38,69 & 38,43 & 49,64 & 46,65 & 128,29 & 121,34 \\
\hline 7. & 56,77 & 56,35 & 30,76 & 30,78 & 22,47 & 22,71 & 73,05 & 73,76 \\
\hline 8 & 54,54 & 63,40 & 40,01 & 48,74 & 29,28 & 28,72 & 64,41 & 58,92 \\
\hline 9 & 64,72 & 65,08 & 3,51 & 3,12 & 4,10 & 3,60 & 116,77 & 115,58 \\
\hline $10 \ldots$ & 63,35 & 63,94 & 9,87 & 7,43 & 13,65 & 10,05 & 137,43 & 135,22 \\
\hline $11 \ldots \ldots$ & 51,94 & 52,51 & 36,37 & 37,10 & 27,26 & 27,48 & 74,97 & 74,08 \\
\hline $12 \ldots \ldots$ & 54,43 & 53,18 & 38,20 & 40,38 & 29,59 & 32,14 & 77,46 & 79,59 \\
\hline $13 \ldots \ldots$ & 67,23 & 73,84 & 6,19 & 6,65 & 5,25 & 4,50 & 84,87 & 67,75 \\
\hline $14 \ldots \ldots \ldots$ & 52,49 & 50,93 & 39,29 & 40,63 & 30,43 & 32,50 & 77,44 & 79,98 \\
\hline $15 \ldots \ldots \ldots \ldots$ & 66,42 & 64,23 & 2,32 & 2,54 & 2,58 & 3,02 & 111,48 & 118,75 \\
\hline $16 \ldots \ldots \ldots \ldots \ldots$ & 64,34 & 64,13 & 2,13 & 2,20 & 2,85 & 2,96 & 133,72 & 134,51 \\
\hline
\end{tabular}

\section{Commentaires}

a) Humidité. Dans neuf cas sur seize l'élimination de la surface conduit à des humidités plus élevées pour l'ensemble du fromage. Mais dans sept cas e'est l'inverse;

b) Matière grasse \% de sec. Dans sept eas il y a à peu près identité entre les deux résultats. Dans six cas l'élimination de la surface se traduit par une augmentation, parfois importante, du taux de matière grasse sur sec. Dans les trois autres, cas c'est l'inverse. Cette observation tient au fait déjà mentionné que la surface des fromages à pâte molle, à l'état frais, est souvent moins grasse que l'intérieur ;

c) Matière sèche totale. La surface étant généralement plus sèche, son élimination conduit à des résultats plus faibles pour la matière sèche totale du fromage entier. C'est ce que l'on observe neuf fois sur seize. Dans deux cas les résultats sont voisins. Dans les cinq autres cas c'est l'inverse que l'on constate ;

d) Matière grasse totale. La surface est souvent moins riche en matière grasse sur sec que l'intérieur; son élimination devrait donc augmenter la matière grasse totale du fromage. Mais, le poids de matière sèche totale du fromage étant également diminué par cette élimination, on assiste à une compensation qui conduit à une matière 
grasse totale plus faible dans la majorité des cas. En fait, l'élimination de la surface produit ce résultat dans huit cas sur seize, alors qu'il y a identité dans quatre cas et que dans les quatre autres cas, on constate l'inverse.

Donc, dans le cas des fromages frais, on constate des résultats irréguliers. L'influence de l'élimination de la surface se traduit tantôt dans un sens, tantôt dans l'autre. Cela se comprend si l'on songe que dans un fromage très frais (le lendemain ou le surlendemain de sa fabrication) la croûte proprement dite n'est pas formée et peut même dans certains cas, représenter lo partie la plus humide du fromage. Néanmoins l'élimination de cette surface se tradvit dans la majorité des eas par une diminution du taux de matière sèche (pour cent et totale) par une augmentation du taux de matière grasse pour cent de sec et par une diminution de la matiére grasse totale.

\section{Fromages demi-affinés.}

\begin{tabular}{|c|c|c|c|c|c|c|c|c|c|}
\hline \multirow{2}{*}{\multicolumn{2}{|c|}{ 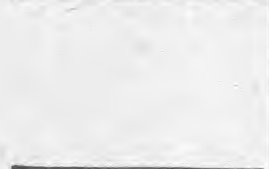 }} & \multicolumn{2}{|c|}{ Humidité \% } & \multicolumn{2}{|c|}{$\begin{array}{l}\text { Matière grasse } \\
\% \text { de sec }\end{array}$} & \multicolumn{2}{|c|}{$\begin{array}{c}\text { Matière grasse } \\
\text { totale }\end{array}$} & \multicolumn{2}{|c|}{$\begin{array}{c}\text { Matiè re sèche } \\
\text { totale }\end{array}$} \\
\hline & & $\begin{array}{l}\text { avec } \\
\text { croûte }\end{array}$ & $\begin{array}{l}\text { sans } \\
\text { cro to }\end{array}$ & $\begin{array}{l}\text { avec } \\
\text { croûte }\end{array}$ & $\begin{array}{l}\text { sans } \\
\text { croûte }\end{array}$ & $\begin{array}{l}\text { avec } \\
\text { croûte }\end{array}$ & $\begin{array}{l}\text { sans } \\
\text { croúte }\end{array}$ & $\begin{array}{l}\text { avec } \\
\text { croûte }\end{array}$ & $\begin{array}{l}\text { sans } \\
\text { croûte }\end{array}$ \\
\hline 1 & $\ldots \ldots \ldots \ldots \ldots$ & 62,33 & 59,74 & 3,95 & 3,92 & 5,48 & 5,81 & 138,62 & 148,15 \\
\hline 2 & $\ldots \ldots \ldots \ldots \ldots$ & 56,39 & 56,00 & 30,79 & 32,43 & 30,75 & 32,67 & 99,86 & 100,76 \\
\hline 3 & $\ldots$ & 52,38 & 51,06 & 30,20 & 35,20 & 31,99 & 38,25 & 105,71 & 108,64 \\
\hline 4 & ............ & 57,11 & 53,61 & 37,42 & 37,27 & 39,16 & 42,18 & 104,65 & 113,19 \\
\hline 5 & $\ldots \ldots \ldots \ldots \ldots$ & 63,81 & 65,31 & 1,46 & 0,66 & 1,17 & 0,51 & 80,34 & 77,01 \\
\hline 6 & ....... & 61,00 & 62,22 & 27,53 & 27,10 & 32,43 & 30,92 & 117,78 & 114,09 \\
\hline 7 & n........... & 54,02 & 51,41 & 35,72 & 33,44 & 34,52 & 34,12 & 96,55 & 102,03 \\
\hline 8 & $\ldots \ldots \ldots \ldots \ldots$ & 56,90 & 57,46 & 35,73 & 38,83 & 44,04 & 47,24 & 123,26 & 121,66 \\
\hline 9 & $\ldots \ldots \ldots \ldots \ldots$ & 52,46 & 52,93 & 35,59 & 36,69 & 43,14 & 44,03 & 121,22 & 120,02 \\
\hline 10 & $\ldots \ldots \ldots \ldots \ldots$ & 49,66 & 50,02 & 36,31 & 39,13 & 19,37 & 20,73 & 53,36 & 52,97 \\
\hline 11 & $\ldots \ldots \ldots \ldots$ & 47,71 & 51,74 & 36,54 & 37,46 & 44,90 & 42,48 & 122,88 & 113,41 \\
\hline 12 & $\ldots \ldots \ldots \ldots$ & 54,43 & 55,54 & 36,05 & 37,15 & 41,40 & 41,63 & 114,83 & 112,03 \\
\hline
\end{tabular}

\section{Commentaires}

a) Humidité. Dans sept cas sur douze l'élimination de la croûte conduit à des résultats plus élevés en ce qui concerne l'humidité. Dans les cinq autres cas c'est l'inverse;

b) Matière grasse \% de sec. Dans sept cas sur douze l'élimination de la croûte entraîne une augmentation. Dans deux cas il y a identité. Dans trois cas seulement il y a diminution. Ce résultat. confirme les précédents ; 
c) Matière sèche totale. Dans sept cas sur douze également l'élimination de la croûte entraîne une diminution du poids de la matière sèche totale. Dans cinq cas, c'est l'inverse ;

d) Matière grasse totale. Dans six cas sur douze l'élimination de la croûte a augmenté le poids de matière grasse totale. Dans trois cas elle n'eut pas d'influence et dans trois cas elle fournit le résultat inverse.

Ces chiffres confirment l'irréyularité de l'incidence de l'elimination de la croûte dans l'analyse des fromages à pâte molle, frais ou même demi-affinés. On conçoit déjà que la logique exigera de conserver cette croûte dans tous les cas.

\section{Fromages affinés.}

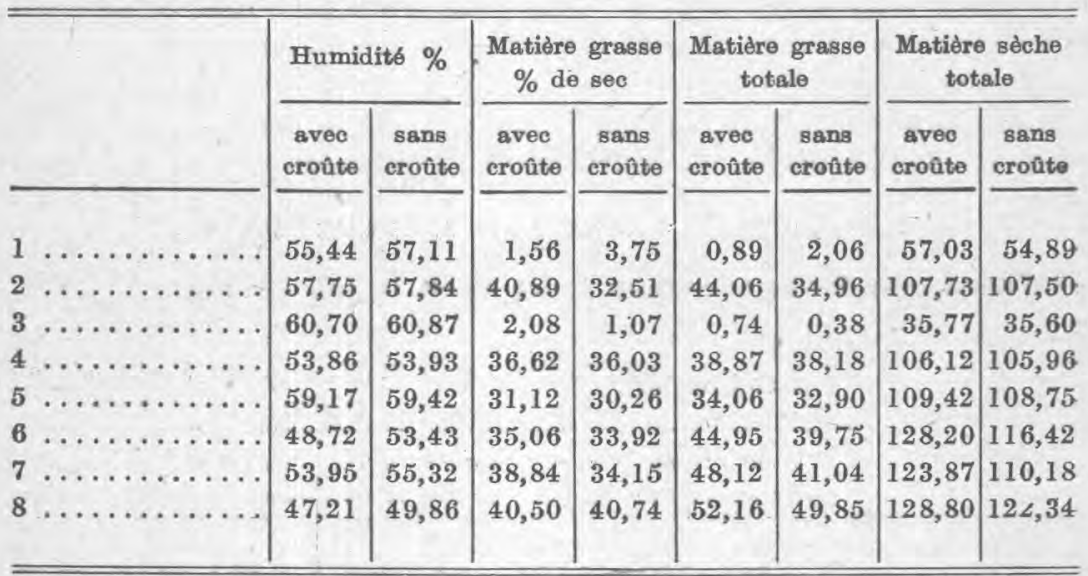

\section{Commentaires}

a) Humidité. Dans tous les cas l'élimination de la croûte conduit, comme il fallait s'y attendre pour des fromages affinés, à des humidités plus élevées. Les différences sont d'ailleurs souvent minimes ;

b) Matière grasse \% de sec. Dans la majorité des cas (six sur huit) l'élimination de la croûte diminue ce pourcentage. Ce résultat est conforme à ce qui a été constaté dans l'analyse séparée des croûtes et de l'intérieur des fromages (croûtes plus riches en matière grasse ramenée au sec);

c) Matière grasse totale. Dans tous les cas (sauf un, correspondant à un fromage maigre) l'élimination de la croûte diminue le poids de matière grasse totale;

d) Matière sèche totale. Dans tous les cas sans exception le poids de matière sèche totale est plus faible lorsque la croûte est éliminèe de l'analyse. 


\section{Conclusions en ce qui concerne les pâtes molles}

$1^{\circ}$ Dans le cas des fromages frais ou demi-affinés l'influence de l'élimination de la croûte ne se fait pas sentir d'une manière régulière. En général (en raison du fait que la croûte est 'souvent plus sèche et moins grasse que l'intérieur) les résultats obtenus pour le fromage entier en éliminant cette croûte tendent à montrer plus d'humidité, plus de matière grasse sur sec, moins de matière sèche totale et une augmentation ou une diminution, suivant les cas, de la matière grasse totale;

$2 \circ$ Dans le cas des fromages affinés les résultats sont plus nets : l'élimination de la croûte entraine dans tous les cas, pour le fromage entier plus d'humidité, moins de matière grasse sur sec (car la croûte des fromages affinés est plus riche que l'intérieur en matière grasse sur. sec), moins de matière grasse totale et moins de matière sèche totale. En d'autres termes l'analyse effectuée sur fromages affinés écroûtés fait perdre au fromage total de la matière grasse et de la matière sèche, en pourcentage et en poids total.

\section{OAS DES fRomages A PATE FERME}

10 Fromages frais (quelques jours après la fabrication).

\begin{tabular}{|c|c|c|c|c|c|c|c|c|c|}
\hline & & \multicolumn{2}{|c|}{ Humidité \% } & \multicolumn{2}{|c|}{$\begin{array}{c}\text { Matière sèche } \\
\% \\
\end{array}$} & \multicolumn{2}{|c|}{$\begin{array}{c}\text { Matière grasse } \\
\text { totale }\end{array}$} & \multicolumn{2}{|c|}{$\begin{array}{c}\text { Matière sêche } \\
\text { totale } \\
\end{array}$} \\
\hline & & $\begin{array}{l}\text { avee } \\
\text { croûte }\end{array}$ & $\begin{array}{c}\text { sans } \\
\text { croûte }\end{array}$ & $\begin{array}{c}\text { avec } \\
\text { croûte }\end{array}$ & $\begin{array}{l}\text { sans } \\
\text { croûte }\end{array}$ & $\begin{array}{l}\text { avec } \\
\text { croûte }\end{array}$ & $\begin{array}{c}\text { sans } \\
\text { eroûte }\end{array}$ & $\begin{array}{c}\text { svec } \\
\text { croûte }\end{array}$ & $\begin{array}{l}\text { sans } \\
\text { croûte }\end{array}$ \\
\hline 1 & 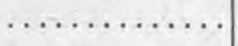 & 44,94 & 47,93 & 16,40 & 14,13 & 10,92 & 8,90 & 66,62 & 63,00 \\
\hline 2 & $\ldots \ldots \ldots \ldots \ldots$ & 45,52 & 46,30 & 34,63 & 30,72 & 67,17 & 58,74 & 193,94 & 191,17 \\
\hline 3 & $\ldots \ldots \ldots \ldots \ldots$ & 43,64 & 45,42 & 37,56 & 36,29 & 48,26 & 45,16 & 128,50 & 124,44 \\
\hline 4 & $\ldots \ldots \ldots \ldots \ldots$ & 55,68 & 55,97 & 15,97 & 14,10 & 17,13 & 15,02 & 107,25 & 106,55 \\
\hline 5 & $\ldots \ldots \ldots \ldots \ldots$ & 49,03 & 49,13 & 33,80 & 35,44 & 27,56 & 28,84 & 81,55 & 81,39 \\
\hline 6 & $\ldots \ldots \ldots \ldots$ & 39,92 & 46,15 & 37,43 & 38,06 & 35,98 & 32,80 & 96,13 & 86,16 \\
\hline 7 & $\ldots \ldots \ldots \ldots$ & 49,24 & 55,31 & 25,78 & 25,66 & 26,04 & 22,62 & 101,01 & 88,93 \\
\hline 8 & $\ldots \ldots \ldots \ldots$ & 39,00 & 47,49 & 33,27 & 32,64 & 31,68 & 26,70 & 95,16 & 81,91 \\
\hline 9 & $\ldots \ldots \ldots \ldots \ldots$ & 46,30 & 47,93 & 32,96 & 33,60 & 20,35 & 20,12 & 61,75 & 59,88 \\
\hline
\end{tabular}

\section{Commentaires}

a) Humidité. L'élimination de la croûte se traduit dans tous les cas, par un plus grand pourcentage d'humidité dans le fromage;

b) Matière grasse \% de sec. Dans six cas sur neuf l'élimination de la croûte entraîne un appauvrissement du fromage en matière grasse. Dans trois cas on observe le résultat inverse ; 
c) Matière grasse totale. Dans tous les cas sauf un, cette élimination diminue le poids de matière grasse totale du fromage (car, comme on l'a vu, la croûte des fromages à pâte ferme est presque toujours plus riche en matière grasse que l'intérieur);

d) Matière sèche totale. Dans tous les cas, l'élimination de la eroûte conduit à une diminution du poids fotal dè matière sèche du fromage - ce qui était également à prévoir puisque la croûte est plus sèche que l'intérieur.

\section{$2^{\circ}$ Fromages demi-affinés.}

\begin{tabular}{|c|c|c|c|c|c|c|c|c|c|}
\hline & & \multicolumn{2}{|c|}{ Humidité \% } & \multicolumn{2}{|c|}{$\begin{array}{c}\text { Matière grasse } \\
\% \text { de sec } \\
\end{array}$} & \multicolumn{2}{|c|}{$\begin{array}{l}\text { Matière grasse } \\
\text { totale }\end{array}$} & \multicolumn{2}{|c|}{$\begin{array}{c}\text { Matière sèche } \\
\text { totale }\end{array}$} \\
\hline & & $\begin{array}{c}\text { avec } \\
\text { croûte }\end{array}$ & \begin{tabular}{|c|} 
sans \\
eroûte
\end{tabular} & $\begin{array}{c}\text { avec } \\
\text { croûte }\end{array}$ & $\begin{array}{l}\text { sans } \\
\text { croûte }\end{array}$ & $\begin{array}{c}\text { avec } \\
\text { cronte }\end{array}$ & $\begin{array}{c}\text { sans } \\
\text { croâte }\end{array}$ & $\begin{array}{c}\text { avec } \\
\text { croûte }\end{array}$ & $\begin{array}{l}\text { sans } \\
\text { croûte }\end{array}$ \\
\hline 1 & 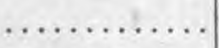 & 51,34 & 55,46 & 19,31 & 19,66 & 22,46 & 20,93 & 116,29 & 106,45 \\
\hline 2 & .... & 50,34 & 56,50 & 23,84 & 22,38 & 21,90 & 18,01 & 91,87 & 80,47 \\
\hline 3 & $\ldots \ldots \ldots \ldots$ & 42,80 & 47,26 & 32,77 & 32,15 & 50,62 & 45,79 & 154,44 & 142,39 \\
\hline 4 & & 53,30 & 55,56 & 15,58 & 15,34 & 17,39 & 16,32 & 111,61 & 106,21 \\
\hline 5 & & 49,67 & 53,90 & 20,08 & 18,26 & 24,64 & 20,20 & 120,79 & 110,64 \\
\hline 6 & $\ldots \ldots \ldots \ldots$ & 52,11 & 56,68 & 16,34 & 18,07 & 13,31 & 13,26 & 81,41 & 73,64 \\
\hline 7 & $\ldots \ldots \ldots \ldots$ & 52,18 & 57,26 & 20,70 & 22,81 & 25,04 & 24,66 & 120,98 & 108,13 \\
\hline 8 & . & 51,24 & 53,96 & 21,30 & 22,98 & 24,93 & 25,39 & 117,02 & 110,49 \\
\hline 9 & 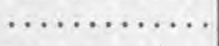 & 46,46 & 48,00 & 34,62 & 32,17 & 29,10 & 26,26 & 84,05 & 81,64 \\
\hline 10 & & 53,26 & 54,82 & 22,14 & 22,06 & 21,11 & 20,33 & 95,34 & 94,16 \\
\hline 11 & $\ldots \ldots$ & 50,63 & 54,07 & 24,68 & 21,57 & 37,17 & 30,22 & 150,57 & 140,08 \\
\hline 12 & & 56,93 & 58,38 & 23,82 & 22,20 & 25,65 & 23,10 & 107,67 & 104,05 \\
\hline 13 & $\ldots \ldots \ldots$ & 54,30 & 57,90 & 21,77 & 20,54 & 27,76 & 24,13 & 127,50 & 117,45 \\
\hline
\end{tabular}

\section{Commentaires}

a) Humidité. Dans tous les cas l'élimination de la croûte entraîne une augmentation du pourcentage d'humidité du fromage ;

b) Matière grasse \% de sec. Dans neuf cas sur treize cette élimination entraîne un appauvrissement du fromage en matière grasse. Dans les quatre autres cas on observe le résultat inverse ;

c) Matiêre grasse totale. Dans tous les cas sauf un, cette élimination diminue le poids de matière grasse totale du fromage et pour les mêmes raisons que précédemment ;

d) Matière sèche totale. Dans tous les cas, l'élimination de la croûte pour l'analyse diminue le poids total de matière sèche du fromage. 


\section{$3^{\circ}$ Fromages affinés.}

\begin{tabular}{|c|c|c|c|c|c|c|c|c|}
\hline & \multicolumn{2}{|c|}{ Humidité \% } & \multicolumn{2}{|c|}{$\begin{array}{c}\text { Matière grasse } \\
\% \text { de sec }\end{array}$} & \multicolumn{2}{|c|}{$\begin{array}{c}\text { Matière grasse } \\
\text { totale }\end{array}$} & \multicolumn{2}{|c|}{$\begin{array}{l}\text { Matière sèche } \\
\text { totale }\end{array}$} \\
\hline & $\begin{array}{l}\text { avec } \\
\text { croûte }\end{array}$ & $\begin{array}{c}\text { sans } \\
\text { croûte }\end{array}$ & $\begin{array}{c}\text { avec } \\
\text { eroûte }\end{array}$ & $\begin{array}{c}\text { sans } \\
\text { eroûte }\end{array}$ & $\begin{array}{c}\text { avec } \\
\text { eroûte }\end{array}$ & $\begin{array}{c}\text { sans } \\
\text { eroûte }\end{array}$ & $\begin{array}{c}\text { avec } \\
\text { eroûte }\end{array}$ & $\begin{array}{l}\text { sans } \\
\text { croûte }\end{array}$ \\
\hline 1 & 47,31 & 51,70 & 20,91 & 19,91 & 19,83 & 17,33 & 94,84 & 86,94 \\
\hline $2 \ldots \ldots \ldots$ & 47,95 & 53,36 & 23,07 & 23,71 & 24,86 & 22,89 & 107,74 & 96,54 \\
\hline $3 \ldots \ldots$ & 34,43 & 43,11 & 39,01 & 38,61 & 25,58 & 21,97 & 65,57 & 56,89 \\
\hline $4 \ldots \ldots$ & 40,48 & 55,08 & 23,38 & 25,60 & 22,41 & 18,51 & 95,82 & 72,32 \\
\hline $5 \ldots$ & 38,49 & 44,81 & 25,54 & 24,91 & 24,19 & 21,17 & 94,72 & 84,99 \\
\hline $6 \ldots \ldots$ & 45,92 & 53,59 & 21,56 & 25,03 & 16,20 & 16,15 & 75,17 & 64,50 \\
\hline $7 \ldots \ldots$ & 47,67 & 55,02 & 16,95 & 14,29 & 14,28 & 10,35 & 84,25 & 72,41 \\
\hline $8 \ldots \ldots$ & 38,93 & 38,29 & 18,92 & 17,89 & 12,13 & 11,59 & 64,12 & 64,79 \\
\hline $9 \ldots$ & 47,70 & 55,73 & 14,81 & 11,52 & 9,53 & 6,27 & 64,32 & 54,45 \\
\hline $10 \ldots \ldots \ldots \ldots$ & 52,88 & 56,15 & 16,38 & 16,48 & 14,82 & 13,88 & 90,47 & 84,19 \\
\hline $11 \ldots \ldots \ldots \ldots$ & 36,94 & 50,67 & 21,91 & 19,78 & 12,71 & 8,97 & 58,01 & 45,38 \\
\hline $12 \ldots \ldots \ldots \ldots \ldots$ & 40,81 & 41,78 & 27,31 & 24,68 & 15,36 & 13,65 & 56,23 & 55,30 \\
\hline $13 \ldots$ & 32,08 & 42,41 & 38,60 & 39,01 & 70,79 & 60,67 & 183,38 & 155,49 \\
\hline $14 \ldots \ldots \ldots \ldots$ & $48,2 i$ & 51,83 & 34,22 & 32,69 & 48,37 & 42,99 & 141,35 & 131,50 \\
\hline $15 \ldots$ & 46,88 & 55,41 & 17,47 & 17,31 & 17,44 & 14,51 & 99,86 & 83,82 \\
\hline $16 \ldots \ldots \ldots$ & 44,84 & 48,82 & 23,36 & 20,92 & 13,79 & 11,45 & 59,02 & 54,76 \\
\hline
\end{tabular}

Commentaires

a) Humidité. Dans tous les cas sauf un, l'élimination de la croûte entraîne une augmentation du pourcentage d'humidité des fromages ;

b) Matière grasse \% de sec. Dans dix cas sur seize l'élimination de la croûte conduit à une teneur en matière grasse plus faible (comme dans la série précédente). Dans deux cas il n'y a pas de différence et dans quatre cas on constate l'effet inverse ;

c) Matière grasse totale. Dans tous les cas, eatte élimination diminue le poids de matière grasse totale du fromage ;

d) Matière sèche totale. Dans tous les cas (sauf un où il y a à peu près similitude) l'élimination de la croûte conduit à un poids de matière sèche totale plus faible.

\section{Conclusion en ce qui concerne les pâtes fermes}

L'élimination de la croûte se traduit par un pourcentage d'humidité trop élevé, quel que soit l'âge du fromage.

En ce qui concerne le taux de matière grasse sur sec, cette élimination entraîne un appauvrissement dans la majorité des cas. Mais on constate aussi le résultat inverse. 
En revanche, en ce qui concerne les éléments totaux (matière grasse totale et matière sèche totale) l'élimination de la croûte entraîne dans tous les cas une perte d'ailleurs irrégulière et quelquefois très importante.

$$
\text { ** } *
$$

RÉSUMÉ ET CONCLUSIONS GÉNÉRALES

\section{DIFFÉRENCES DE COMPOSITION ENTRE LA CROUTE ET L'INTÉRIEUR DES FROMMAES}

\section{Dans le cas des pâtes molles.}

a) Humidité. Dans les fromàges jeunes la surface est tantôt plus humide, tantôt plus sèche que l'intérieur. Dans les fromages affinés la croûte est toujours plus sèche que l'intérieur ;

b) Matière grasse \% de sec. Dans les fromages jeunes la surface est souvent moins grasse que l'intérieur (matière grasse brûlée par les moisissures) ;

Dans les fromages affinés la surface est souvent plus grasse relativement que l'intérieur, par suite d'une déperdition superficielle de matière sèche.

\section{$2^{\circ}$ Dans le cas des pâtes fermes.} térieur ;

a) Humidité. Dans tous les cas la croûte est plus sèche que l'in-

b) Matière grasse \% de sec. Dans la majorité des cas (et quel que soit l'âge du fromage) la croûte est plus riche relativement que l'intérieur (déperdition superficielle de la matière sèche).

\section{INFLueNCE dE L'ELIMINATION DE LA CROUTE SUR LES RÉSULTATS DE L'ANALYSE}

\section{Dans le cas des pâtes molles.}

Dans les fromages frais ou demi-affinés cette élimination entraîne des résultats irréguliers, ne permettant pas de conclusion.

Dans les fromages affinés l'élimination de la eroûte entraîne une diminution de la matière sèche $\%$, une diminution (en général) de la matière grasse $\%$ de sec et une diminution certaine dans tous les cas de la matière sèche totale et de la matière grasse totale.

\section{Dans le cas des pâtes fermes.}

Dans tous les cas l'élimination de la croûte diminue le taux de matière sèche $\%$.

Dans la majorité des cas elle entraîne une diminution de la matière grassa $\%$ de sec. Mais ce résultat n'est pas général. On constate l'effet inverse quel que soit l'âge du fromage. 
Enfin, dans tous les cas, l'élimination de la croûte entraîne une diminution parfois très importante de la matière sèche totale et de la matière grasse totale.

\section{Conclusion}

L'élimination de la croûte des fromages entraîne dans l'analyse rapportée au fromage entier, une perte de matière sèche en pourcentage et en quantité totale et une perte de matière grasse totale (la perte de matière grasse \% de sec n'est pas absolument générale).

Ces conclusions sont valables pour les pâtes pressées quel que soit leur âge et pour les pâtes molles affinées. (Dans le cas des pâtes molles jeunes, les résultats sont irréguliers).

Done en éliminant cette croûte on commet des erreurs parfois très importantes et c'est pourquoi il est indispensable, dans tous les cas, d'effectuer l'analyse sur l'échantillon entier, croûte comprise.

(La partie expérimentale de ce travail a été réalisée avec la eollaboration de M. Frnck du Laboratoire de Chimie de la Laiterie des Fermiers Réunis.)

\section{LE COLOSTRUM DE VACHE}

\section{Composition - Propriétés}

REPERCUSSIONS EN INDUSTRIE LAITIERE

par

\section{A. HOUDINIËRE}

\section{Ingénieur agricole}

Docteur-Vétérinaire sanitaire du département de la Seine

(suite)

\section{B. CONSTANTES PHYSIQUES ET OHIMIQUES DU COLOSTRUM}

L'étude de la constitution physico-chimique du colostrum appelle celle de ses constantes physiques et chimiques.

On peut classer celles-ci en deux catégories :

Les constantes fixes. - Ce sont celles qui ne sont pas ou peu influencées au cours de la période colostrale par les variations de la constitution physico-chimique. Tel est l'exemple typique de l'abaissement du point de congélation.

Les constantes variables. - Il s'agit des constantes auxquelles la constitution physico-chimique imprime des variations notables au cours de la période colostrale, mais qui, toutefois restent entre des limites fixes. Celles-ci sont définies, au début de la période colostrale, par les constantes du premier colostrum et à la fin de cette 